\title{
The Use of Caudal/Epidural Catheter as the Primary Anesthetic with Dexmedetomidine Secondary to High Risk of Post-Operative Apnea in Premature Infants
}

\author{
Joel Waring, Alyssa Lowenwirt, Miriam Flaum, Eduard Logvinskiy, Dennis E. Feierman \\ Department of Anesthesiology, Maimonides Medical Center, Brooklyn, NY, USA \\ Email:dfeierman@maimonidesmed.org
}

Received 4 March 2016; accepted 18 April 2016; published 21 April 2016

Copyright $@ 2016$ by authors and Scientific Research Publishing Inc.

This work is licensed under the Creative Commons Attribution International License (CC BY).

http://creativecommons.org/licenses/by/4.0/

c) (i) Open Access

\begin{abstract}
Life threatening post-operative apnea is a known complication of general anesthesia in premature infants. Neuraxial anesthesia has been associated with reduced risk of post-operative apnea. We report two cases, presenting for bilateral inguinal hernia repair, where we used a caudal/epidural catheter for the neuraxial block combined with dexmedetomidine for sedation in preterm infants at high risk for postoperative respiratory complications. Dexmedetomidine assisted in providing both favorable surgical conditions via its well proven sedative effects without respiratory comprised and decreased the discomfort and stress to the child during placement of the epidural catheter and the neuraxial anesthesia provided excellent analgesia and surgical conditions.
\end{abstract}

Keywords

Prematurity, Apnea, Post-Operative Complication

\section{Introduction}

Improvements in neonatal intensive care have allowed more premature infants to survive birth at lower post conceptual age [1]. As such, more infants are presenting for surgery at extremely young ages. Among these surgeries, the inguinal hernia repair is very common in both pre-term and former pre-term infants [2]. Among the anesthetic concerns, post-operative apnea is a major potential complication when performing hernia repairs in these patients. General anesthesia is associated with an increased risk of apnea [3]; therefore, regional anesthesia is an excellent alternative for this patient population. The reported incidence of post-operative respiratory dys- 
function after general anesthesia is high. Life threatening postoperative apnea is a known complication of general anesthesia in young infants that are premature. This risk is even greater in infants that are anemic or less than 60 weeks post gestational age. Neuraxial anesthesia has been shown to reduce the risk of apnea [4]. Furthermore, performing epidural anesthesia through a caudal catheter has proven to be beneficial for inguinal hernia repair since one can easily increase in duration and degree of anesthesia with additional incremental dosing [5]. One problem with regional techniques is that an awake infant can make the surgery very difficult or impossible without sedation: i.e., crying and bearing down can cause the abdominal contents to herniate through the incision. This report describes the successful ability of two premature infants who had undergone bilateral inguinal hernia surgery with a caudal/epidural catheter and sedation with dexmedetomidine, an agent that has little respiratory effects [6].

\section{Case}

Written consent to publish this case report from the parent was obtained for the first child. Multiple attempts to contact the second patients' parents were not successful. Thus we sought approval from our local Institutional Review Board who granted permission to publish this case report. Both infants presented for bilateral inguinal hernia repair. First neonate was 45 weeks post conceptual age, $2 \mathrm{~kg}$ infant (born at 28 weeks gestation via emergency C-section) with history of respiratory distress syndrome (intubated $\times 1$ month), patent ductus arterious, retinopathy of prematurity, anemia of prematurity, reflux, osteopenia and episodes of apnea. Second neonate was 40 weeks post conceptual age, $2.2 \mathrm{~kg}$ infant (born at 28 week gestation via vaginal delivery) with history of chronic lung disease, reflux, retinopathy of prematurity, anemia of prematurity and history of apnea s/p treatment with caffeine. In both cases, a decision was made to proceed with caudal/epidural catheter as the primary anesthetic secondary to high risk of post-operative apnea. Additionally, we opted to use dexmedetomidine for sedation to provide calm, non-crying infant to facilitate the surgery and because favorable lack of it respiratory effects [6]. During examination of patient's sacrum, no dimples were found in either child or any contraindications for caudal anesthesia. Glycopyrrolate $0.02 \mathrm{mg}$ IV $(10 \mathrm{mcg} / \mathrm{kg})$ was given for its antisialagogue and antimuscarinic effects and for the ability of this class of drugs to increase the sensitivity to detect inadvertent IV injections of local anesthetics compounded with epinephrine [7]. After sterile preparation with betadine and draping, an $18 \mathrm{~g}$ angiocatheter needle was advanced through the sacral hiatus. Aspiration was negative for blood or CSF. A $20 \mathrm{~g}$ caudal catheter was threaded via $18 \mathrm{~g}$ angiocatheter to the level of the L1-2 vertebral interspace and secured in a sterile manner. The distance from the sacral hiatus to the L1 spinous process was estimated by measuring the outside length from the angiocatheter to the L1-2 interspace. $1 \mathrm{ml}$ of Bupivacaine $0.5 \%$ (approximately $2.5 \mathrm{mg} / \mathrm{kg}$ ) with $5 \mathrm{mcg} / \mathrm{ml}$ of epinephrine was injected through the caudal/epidural catheter. One mcg of dexmedetomidine $(0.5 \mathrm{mcg} / \mathrm{kg})$ was given IV, followed by another microgram five minutes later. The dexmedetomidine was given after the epidural placement for the first child and before the epidural for the second in an attempt to decrease the discomfort and stress to the child during placement of the epidural catheter. Neuraxial anesthesia provided excellent analgesia, surgical conditions and post operative analgesia. The dexmedetomidine provided a quiet calm baby, augmenting the favorable surgical conditions. At the end of the procedure, caud$\mathrm{al} /$ epidural catheter was removed, with intact tip in both cases. The infants were transfer back to the NICU for monitoring, which included EKG, respiratory monitoring and pulse oximetry. Both infants had uncomplicated post-operative courses without any observed apnea episodes.

\section{Discussion}

There is some controversy as to the most appropriate time to perform a hernia repair. Due to an increase in risk of strangulation, incarceration, and testicular atrophy some surgeons support surgical hernia repair during the initial hospitalization [2]. This claim is supported by a study performed by Lautz et al. 1123 premature neonates, who had undergone inguinal repair, were evaluated. They found that incarceration occurred in $16 \%$ of those that had the surgery during the initial hospitalization visit [8]. Additionally, of the premature neonates that underwent hernia repair beyond 40 weeks post conceptual age (PCA), 21\% had incarceration, as opposed to 9\% who had the surgery between 36 to 39 weeks PCA. The risk of incarceration had more than doubled when delaying surgery, suggesting that surgery should not be delayed.

Postoperative apnea is another risk when performing inguinal hernia repair in premature infants. Apnea of prematurity is a common problem that occurs in most premature infants who are born at a gestational age less 
than 33 weeks old. Apnea of prematurity is a developmental disorder caused by a lack of respiratory control. Infants with apnea of prematurity have pauses in breathing for over 15 - $20 \mathrm{~s}$ that may cause oxygen desaturation and bradycardia [9]. Apnea in infants can lead to deleterious effects on neurological development due to anoxia of the CNS [10]. General anesthesia increases the risk of apnea [3] [11]. Of the 47 preterm infants $<60$ weeks PCA studied by Kurth et al., 18 preterm infants had prolonged apnea (>15 s) and 7 had short apnea (6 - 15 s) postoperatively [11]. PCA has an inverse relationship with apnea; as PCA decreases, incidence of apnea increases: a short PCA is a strong predictor of apnea [10] [12]. Therefore, post-operative apnea is a major concern for premature infants undergoing hernia surgery.

In a recent study, neonates were randomized to receive either general anesthesia or spinal anesthesia. The spinal anesthesia group had a reduced risk of early postoperative apnea, oxygen desaturation, and level of intervention for apnea [4]. Therefore, utilizing regional anesthesia has clear benefits in premature infants when compared with general anesthesia. Spinal anesthesia has its shortcomings. It can be technically difficult and can be variable both in density and duration. We present an alternative that the authors feel is beneficial when compared to a spinal. Performing epidural anesthesia through caudal catheter is advantageous for premature infants undergoing hernia repair because it allows for incremental doses of anesthetic to be given in order for the anesthetic to reach the appropriate segmental level and/or to increase the duration or action [5]. Furthermore an epidural placed via caudal access maybe a technically easier and more reliable procedure than placement of an intrathecal block. The addition of dexmedetomidine even enhances the anesthetic. Dexmedetomidine may assist in alleviating the stress on the child via its well proven sedative effects. Additionally, a calm and quite baby leads to a quiet and still operative field (crying could make it difficult for the surgeon to reduce the hernias). The use of dexmedetomidine for sedation, with its unique pharmacologic profile of providing sedation without respiratory depression, makes it the ideal drug in patients that are at significant risk for respiratory compromise. It should be noted that neither of the babies had apneic events postoperatively. A search of the literature shows this is the first presentation of the use of the combination of a regional technique in combination with dexmedetomidine in premature infants.

\section{Conclusion}

It is clear that post-operative cardiorespiratory complications occur frequently in former pre-term infants after relatively minor surgical procedures. In the experienced hands of an anesthesiologist, caudal/epidural anesthesia can be safely and reliably performed in high risk infants for inguinal hernia repair, without compromising patient safety and comfort. Dexmedetomidine may assist in providing both favorable surgical conditions and decreasing the discomfort and stress to the child during placement of the epidural catheter, as well as alleviating the stress on the child via its well proven sedative effects.

\section{References}

[1] Rescorla, F.J. and Grosfeld, J.L. (1984) Inguinal Hernia Repair in the Perinatal Period and Early Infancy: Clinical Considerations. Journal of Pediatric Surgery, 19, 832-837. http://dx.doi.org/10.1016/S0022-3468(84)80379-6

[2] Raveenthiran, V. (2014) Controversies Regarding Neonatal Inguinal Hernia. Journal of Neonatal Surgery, 3, 31.

[3] Bouchut, J.C., Dubois, R., Foussat, C., Moussa, M., Diot, N., Delafosse, C., Claris, O. and Godard, J. (2001) Evaluation of Caudal Anaesthesia Performed in Conscious Ex-Premature Infants for Inguinal Herniotomies. Paediatric Anaesthesia, 11, 55-58. http://dx.doi.org/10.1046/j.1460-9592.2001.00617.x

[4] Davidson, A.J., Morton, N.S., Arnup, S.J., de Graaff, J.C., Disma, N., Withington, D.E., Frawley, G., Hunt, R.W., Hardy, P., Khotcholava, M., von Ungern Sternberg, B.S., Wilton, N., Tuo, P., Salvo, I., Ormond, G., Stargatt, R., Locatelli, B.G. and McCann, M.E. (2015) General Anesthesia Compared to Spinal Anesthesia C: Apnea after Awake Regional and General Anesthesia in Infants: The General Anesthesia Compared to Spinal Anesthesia Study-Comparing Apnea and Neurodevelopmental Outcomes, A Randomized Controlled Trial. Anesthesiology, 123, 38-54.

[5] Peutrell, J.M. and Hughes, D.G. (1993) Epidural Anaesthesia through Caudal Catheters for Inguinal Herniotomies in Awake Ex-Premature Babies. Anaesthesia, 48, 128-131. http://dx.doi.org/10.1111/j.1365-2044.1993.tb06850.x

[6] Venn, R.M., Hell, J. and Grounds, R.M. (2000) Respiratory Effects of Dexmedetomidine in the Surgical Patient Requiring Intensive Care. Critical Care (London, England), 4, 302-308. http://dx.doi.org/10.1186/cc712

[7] Sethna, N.F., Sullivan, L., Retik, A., McGowan, F.X., Di Canzio, J. and Zurakowski, D. (2000) Efficacy of Simulated Epinephrine-Containing Epidural Test Dose after Intravenous Atropine during Isoflurane Anesthesia in Children. Re- 
gional Anesthesia and Pain Medicine, 25, 566-572. http://dx.doi.org/10.1097/00115550-200011000-00004

[8] Lautz, T.B., Raval, M.V. and Reynolds, M. (2011) Does Timing Matter? A National Perspective on the Risk of Incarceration in Premature Neonates with Inguinal Hernia. Journal of Pediatrics, 158, 573-577. http://dx.doi.org/10.1016/j.jpeds.2010.09.047

[9] Zhao, J., Gonzalez, F. and Mu, D. (2011) Apnea of Prematurity: From Cause to Treatment. European Journal of Pediatrics, 170, 1097-1105. http://dx.doi.org/10.1007/s00431-011-1409-6

[10] Deykin, E., Bauman, M.L., Kelly, D.H., Hsieh, C.C. and Shannon, D. (1984) Apnea of Infancy and Subsequent Neurologic, Cognitive, and Behavioral Status. Pediatrics, 73, 638-645.

[11] Kurth, C.D., Spitzer, A.R., Broennle, A.M. and Downes, J.J. (1987) Postoperative Apnea in Preterm Infants. Anesthesiology, 66, 483-488. http://dx.doi.org/10.1097/00000542-198704000-00006

[12] Cote, C.J., Zaslavsky, A., Downes, J.J., Kurth, C.D., Welborn, L.G., Warner, L.O. and Malviya, S.V. (1995) Postoperative Apnea in Former Preterm Infants after Inguinal Herniorrhaphy. A Combined Analysis. Anesthesiology, 82, 809822. http://dx.doi.org/10.1097/00000542-199504000-00002 